\title{
Phrenic Nerve Paralysis as the Initial Presentation in Pleural Sarcomatoid Mesothelioma
}

\author{
Go Makimoto ${ }^{a, c}$ d Keiichi Fujiwara ${ }^{a}$ Nobukazu Fujimoto ${ }^{c}$ \\ Ichiro Yamadori $^{\mathrm{b}}$ Toshio Sato $^{\mathrm{a}}$ Takumi Kishimoto $^{c}$ \\ Departments of ${ }^{a}$ Respiratory Medicine and ${ }^{b}$ Laboratory Medicine, National Hospital \\ Organization Okayama Medical Center, 'Department of Respiratory Medicine, Okayama \\ Rosai Hospital, and ${ }^{\mathrm{d}}$ Department of Allergy and Respiratory Medicine, Okayama \\ University Hospital, Okayama, Japan
}

\section{Key Words}

Malignant pleural mesothelioma $\cdot$ Localized sarcomatoid mesothelioma $\cdot$ Phrenic nerve paralysis · Immunohistological examination

\begin{abstract}
A 74-year-old man was referred to our hospital because of persistent cough. A chest radiograph revealed an elevation of the right diaphragm. Computed tomography (CT) images revealed a small nodule localized on the right mediastinum. Five months later, the nodule had grown and was diagnosed as malignant pleural mesothelioma (MPM) by a CTguided needle biopsy. The patient underwent combined chemotherapy, but the disease progressed rapidly and he passed away. On autopsy, microscopic findings and immunohistological examinations supported the diagnosis of sarcomatoid mesothelioma. Therefore, we diagnosed this rare case as localized sarcomatoid MPM showing phrenic nerve paralysis as an initial presentation.

(c) 2014 S. Karger AG, Basel
\end{abstract}

\section{Introduction}

Malignant pleural mesothelioma (MPM) is a disease with poor prognosis. The median survival period is 7.7 months [1], and only an extrapleural pneumonectomy at an early stage can cure the disease. However, early detection of MPM is usually difficult, because its symptoms are nonspecific. In this study, we report a case of sarcomatoid MPM initially presenting as phrenic nerve paralysis. 
Makimoto et al.: Phrenic Nerve Paralysis as the Initial Presentation in Pleural Sarcomatoid Mesothelioma

\section{Case Report}

A 74-year-old man was referred to our hospital because of persistent cough in October 2011. He had a history of prostate enlargement and gastric ulcer. He worked in the shipyard and had been exposed to asbestos for several years during his early twenties. He previously smoked 20 cigarettes per day for 24 years until he stopped smoking at the age of 44 years. A chest radiograph revealed an elevation of the right diaphragm (fig. 1a). Computed tomography (CT) images revealed a small nodule localized on the right mediastinum adjacent to the pericardial cavity (fig. 1b). Five months later, the nodule had developed into a massive tumor (fig. 1c). Finally, MPM was pathologically diagnosed by a CT-guided needle biopsy. The patient underwent combined chemotherapy with carboplatin and pemetrexed, but the disease progressed rapidly and caused superior vena cava syndrome. The patient then underwent palliative thoracic irradiation, but he passed away in June 2012. His family provided consent to conduct an autopsy.

On autopsy, a whitish solid tumor was detected on the right side of the mediastinum, and the tumor had infiltrated the parietal pleura, diaphragm, pericardial cavity, and aorta. The tumor had adhered strongly to the right upper lobe of the lung. Microscopically, the tumor consisted of spindle-shaped cells and collagen fibers, and had necrotic areas (fig. 2). Immunohistochemical examination revealed that the tumor cells were positive for calretinin, Wilms' tumor protein (WT-1), D2-40 and cytokeratin (AE1/AE3 and CAM 5.2), and negative for carcinoembryonic antigen (CEA) and thyroid transcription factor (TTF-1) (fig. 3). Diagnosis of sarcomatoid MPM was confirmed on the basis of these findings.

\section{Discussion}

MPM is classified into 2 types: diffused and localized. Localized MPM is uncommon and presents as a microscopic tumor characterized by a sharp circumscription of the serosal membrane without any evidence of diffused spread [2]. In the present case, a small localized tumor on the right chest wall was detected in October 2011. We suspected that the right phrenic nerve was involved with the tumor resulting in right phrenic nerve paralysis. At the beginning of the course, the lesion was localized. To our knowledge, this is the first report of MPM presenting phrenic nerve paralysis as an initial manifestation.

In order to confirm the diagnosis of sarcomatoid MPM, it is essential to distinguish it from lung sarcomatoid carcinoma (LSC). LSC is defined as an epithelial carcinoma consisting of spindle- or polygonal-shaped tumor cells, and is often a combination of the characteristics of adenocarcinoma or squamous cell carcinoma. It is difficult to differentiate sarcomatoid MPM from LSC; however, immunohistochemistry has proven to be useful in this regard [3]. Takeshima et al. [4] reported that the well-known mesothelial marker D2-40 was useful in differentiating sarcomatoid MPM from the sarcomatoid component of LSC. In our case, tumor cells were positive for both mesenchymal and epithelial markers, including D2-40, and negative for CEA and TTF-1. In addition, the radiological findings in the current case indicated that the primary lesion was apparently not in the lung. Therefore, a final diagnosis of MPM could be ascertained.

In conclusion, we report here a case of localized sarcomatoid MPM showing phrenic nerve paralysis as an initial presentation. It is important to perform immunohistochemical examination with sufficient materials to diagnose sarcomatoid MPM. 


\section{Case Reports in Oncology}

\begin{tabular}{l|l}
\hline Case Rep Oncol 2014;7:389-392 \\
\hline DOI: $10.1159 / 000363760$ & $\begin{array}{l}\text { C 2014 S. Karger AG, Basel } \\
\text { www.karger.com/cro }\end{array}$ \\
\hline
\end{tabular}

Makimoto et al.: Phrenic Nerve Paralysis as the Initial Presentation in Pleura Sarcomatoid Mesothelioma

\section{Disclosure Statement}

The authors state that they have no conflict of interest.

\section{References}

1 Gemba K, Fujimoto N, Aoe K, et al: Treatment and survival analyses of malignant mesothelioma in Japan. Acta Oncol 2013;52:803-808.

-2 Allen TC, Cagle PT, Churg AM, et al: Localized malignant mesothelioma. Am J Surg Pathol 2005;29:866-873.

-3 Kushitani K, Takeshima Y, Amatya VJ, et al: Differential diagnosis of sarcomatoid mesothelioma from true sarcoma and sarcomatoid carcinoma using immunohistochemistry. Pathol Inter 2008;58:75-83.

-4 Takeshima Y, Amatya VJ, Kushitani K, et al: Value of immunohistochemistry in the differential diagnosis of pleural sarcomatoid mesothelioma from lung sarcomatoid carcinoma. Histopathology 2009;54:667-676.
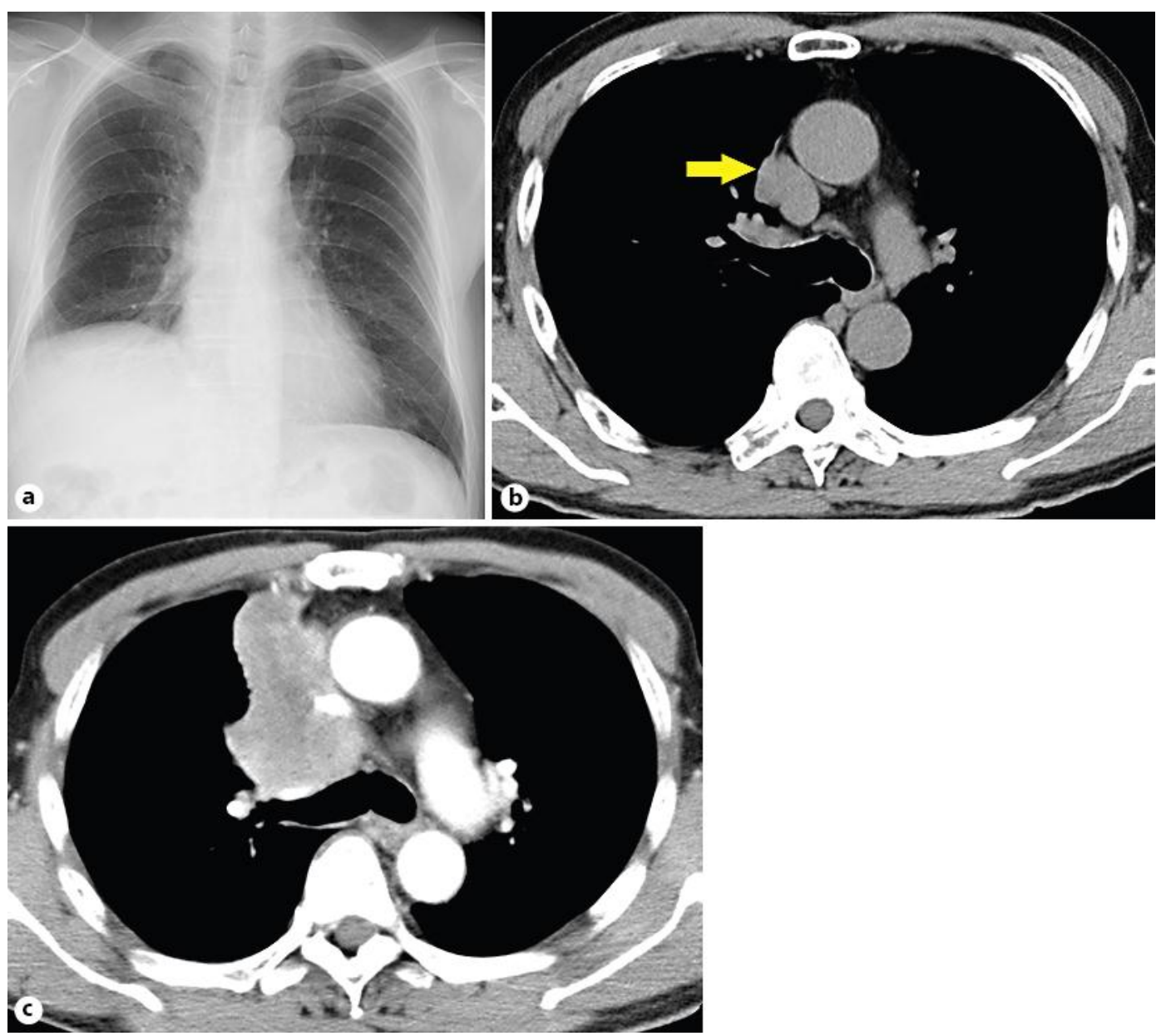

Fig. 1. A chest radiograph taken in October 2011 revealed elevation of the right diaphragm (a). CT images revealed a small nodule localized on the right mediastinum adjacent to the pericardial cavity (b). The nodule had developed into a massive tumor in March 2012 (c). 


\section{Case Reports in Oncology}

\begin{tabular}{l|l}
\hline \multicolumn{2}{l}{ Case Rep Oncol 2014;7:389-392 } \\
\hline DOI: 10.1159/000363760 & $\begin{array}{l}\text { C 2014 S. Karger AG, Basel } \\
\text { www.karger.com/cro }\end{array}$ \\
\hline
\end{tabular}

Makimoto et al.: Phrenic Nerve Paralysis as the Initial Presentation in Pleural Sarcomatoid Mesothelioma

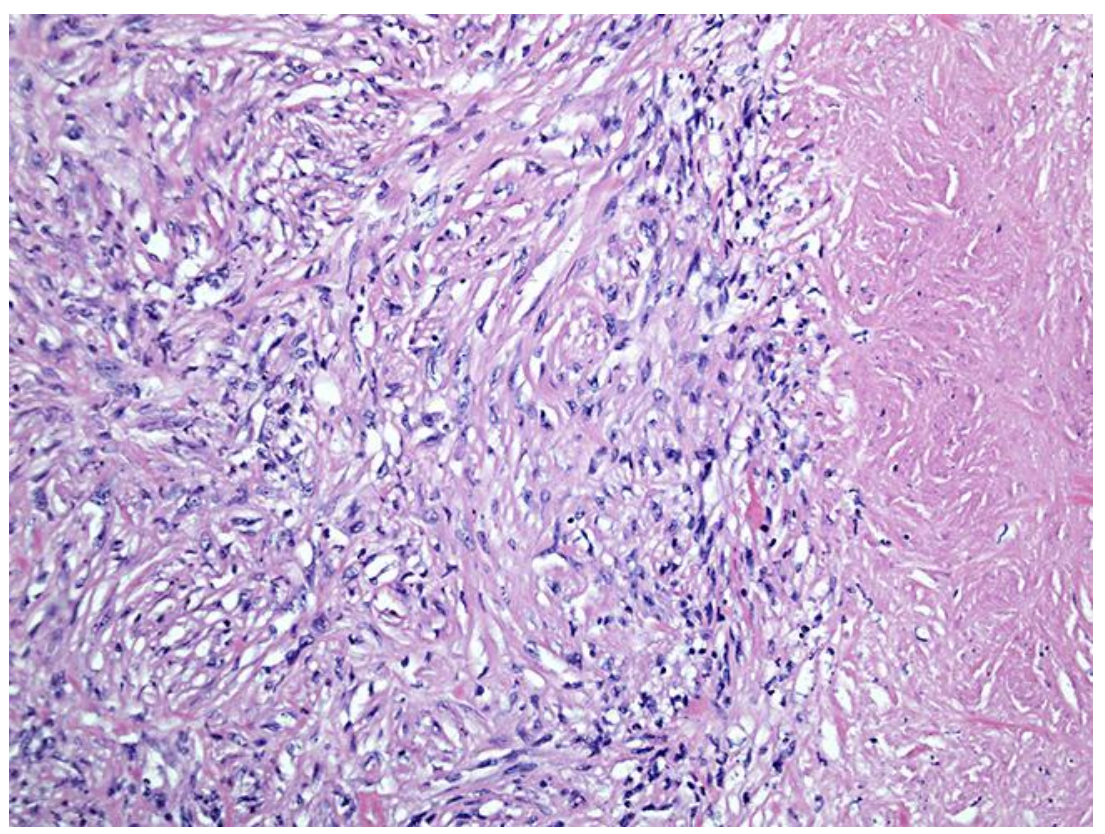

Fig. 2. During autopsy, histopathology of the mediastinal tumor revealed proliferation of spindle-shaped cells and collagen fibers with necrotic areas (hematoxylin and eosin stain).

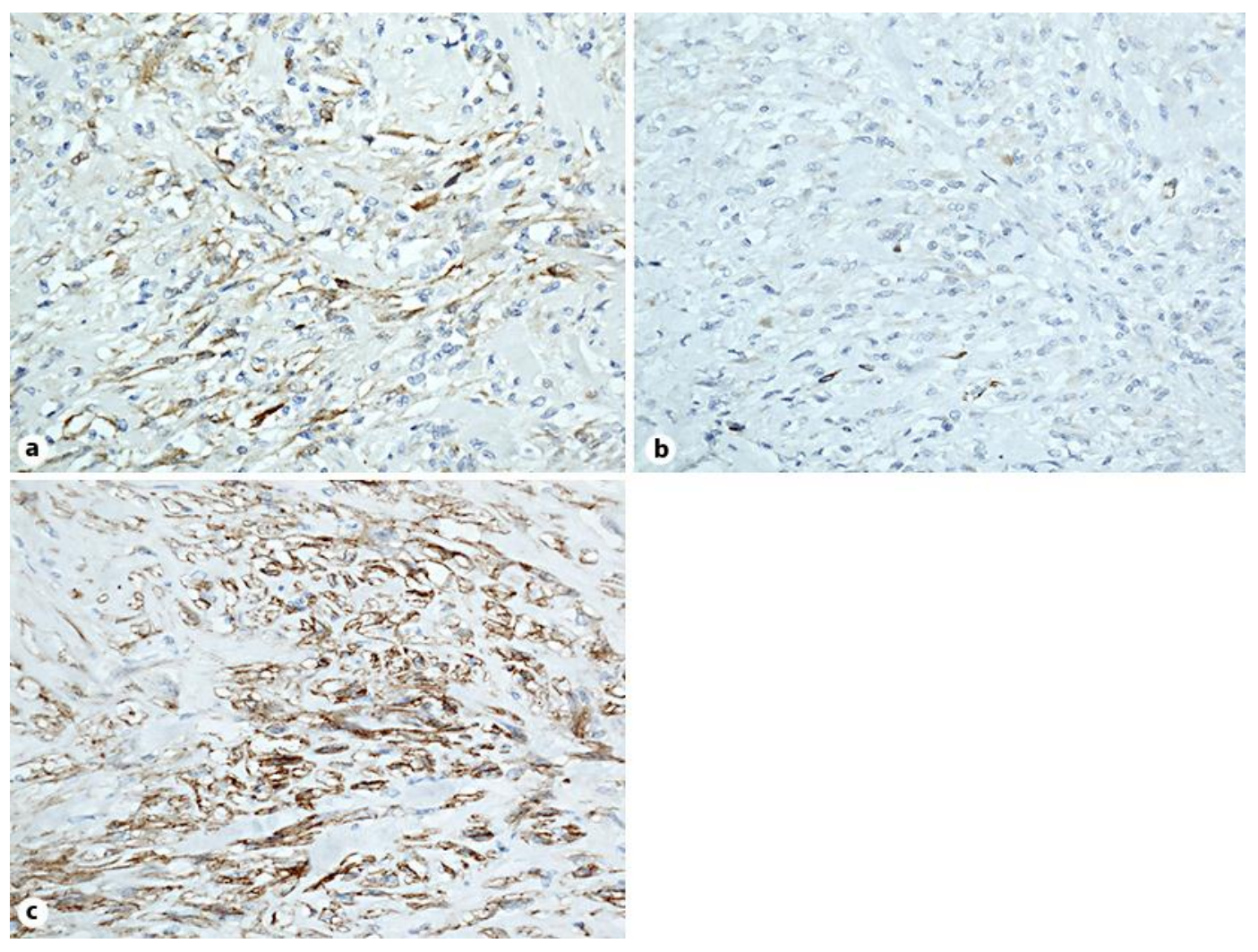

Fig. 3. Immunohistology revealed positive staining for calretinin (a), WT-1 (b), and D2-40 (c). 